\title{
Design and Development of a Cartesian Robot for PCB Drilling: A Systematic Mechatronic Approach
}

\author{
${ }^{1}$ Dera Humphrey N, ${ }^{1}$ Kapuya Engelbert T, ${ }^{1}$ Mlambo Powell, ${ }^{1}$ Chihota Kelvin, ${ }^{1}$ Chiweshe Eugenia R, ${ }^{1}$ Chipuri Ian \\ ${ }^{1}$ Department of Mechatronic Engineering \\ Chinhoyi University of Technology \\ Chinhoyi, Zimbabwe \\ humphrey.dera@gmail.com
}

\begin{abstract}
Design of mechatronic products is a complex process which involves various engineering domains [1] [2] [3]. Choice of an appropriate systematic procedure for product design and development is important in mechatronic system design. The V model (VDI 2206) for mechatronic system design and the Pahl and Beirtz design methodology was suggested for the development of a Cartesian robot for Printed circuit board (PCB) drilling [2]. The system was subdivided into, mechanical, electronics and software engineering domains. Methods suitable for design in each domain were used, and sub systems were developed which then were integrated to develop the whole system. Software that extracts the coordinates of the holes from the gabber file was developed. The file of coordinates will then be transferred to the control unit which will then coordinated the actuators to move to the desired Cartesian coordinates and perform the drilling operation. A graphical user interface was developed also to input the coordinates manually. The designed Cartesian robot is a portable desk machine that can be used to perform the drilling operations. It can also be used as a teaching aid in the computer aided manufacturing courses to demonstrate the tool motion activities.
\end{abstract}

Keywords: Cartesian robot, printed circuit board, mechatronic system design methodology, VDI guideline 2206, Pahl and Beitz design methodology

\section{Introduction}

PCB fabrication is a process which involves a lot of serial steps which are; PCB layout design, routing, drilling of holes and then placement of components and soldering. Various methods are used which range from manual to semiautomated and fully automated PCB fabrication. Due to prohibitive costs of automated PCB fabrication machines most research institutions and academic institution in Zimbabwe depends on the manual process. This process involves chemical etching and then drilling of holes. Drilling of holes can be strenuous, and can result in some misaligned holes which makes population of the PCB difficult and this will lengthen the development process.

Automated CNC machines can be employed to do the PCB drilling with precision and less time, however the cost of buying one is prohibitive [4] especially in research institutions. Available $\mathrm{CNC}$ machines require an expert to develop a code (G codes) for the drilling of the PCB [1]. Most CAD software for PCB layout design however produce a gabber file which contains the coordinates of the holes in the $x-y$ plane. A system that automatically converts that file into usable data can reduce the level of expertise required to formulate the code for machining. This initiative will reduce the process time by the user in producing a product.

A PCB can only be worked in the x-y plane with a single fixed displacement in the $\mathrm{z}$ axis. Developing a robotic system that works within the space defined will reduce the number of axis to 3 . This will reduce the material requirements of the system thereby reducing on the cost of developing the system.

A Cartesian robot can be employed to do the job. Development of such a system borrows knowledge from various engineering domains; mechanical, electrical and information systems. This qualifies the system as a multi domain product and a mechatronic product. This product therefore requires a specialized methodology for the development of the product. Methodologies for mechatronic systems which clearly divides the development work into its respective domains and makes the process more transparent [1] [5] are required for the development of such a system. The V model (VDI 2206 guidelines) was suggested as the methodology for the development of the Cartesian robot.

The developed product can also be used as a teaching aid for students taking courses in Computer Aided Design and Computer Aided Manufacturing. Tool motion can be demonstrated which will give students an appreciation of CNC machines. The product can also be used to demonstrate the application of the $\mathrm{V}$ model in the development of mechatronic systems.

\section{Objectives}

- To design the Cartesian robot chassis that will be able to position the tool piece to the required coordinates. ACRID 2017, June 20-21, Victoria Falls, Zimbabwe

Copyright $(\mathcal{2} 2017$

DOI 10.4108/eai.20-6-2017.2270836 
- To develop the electronic control system that will guide the movement of the tool piece.

- To develop a computer based algorithm that extracts the hole coordinates from the gabber file and process them into usable coordinates for the Cartesian robot

- To integrate the systems into a single system that can drill holes on PCBs.

\section{Methodology}

PCB drilling requires precision in hole identification and drilling so that the holes will be aligned well for to ensure easy placement of ICs. Employing a robot to do the task will be ideal. Development of such a system will borrow knowledge from various engineering disciplines there by making it a multidisciplinary system. For this reason, a methodology that supports multidisciplinary approach was required. V model (VDI 2206 guidelines) was used to come up with a process model for the development of a Cartesian robot for PCB drilling. The V model will aid in dividing development work into respective engineering domains and this makes the process more transparent and organized. It also provides a clear structure of the development process from one level to the next [1]. This will enhance a systematic and organized procedure or development process of the Cartesian robot.

The process model starts with development of a requirement list for the Cartesian robot. Data was gathered, through guided observations. The data was used to characterize the problem at hand. From the problem characterization, a set of narrative requirements was developed. The Pahl and Beitz requirements checklist [5] was used together with the initial requirements list to formulate the design specifications of the Cartesian robot.

A principle solution which addresses all the requirements was developed. The principle solution shows how the system as a whole will work. The principle solution was sub divided into its respective Engineering domains, namely Mechanical and electrical engineering. The electrical engineering domain combined the electronics control and software development. An analysis in the respective domains was made based on the basic physical principles involved. In each domain, various solutions were suggested and the best solution was recommended for each domain resulting in the development of design modules in each domain.

The modules were further broken down to develop the respective parts that are required to develop each module. The parts were designed and sized resulting in the development of detailed drawings for each domain. From the detailed drawings, a bill of materials was generated and the required materials were ordered.

Fabrication and testing of parts was done. The parts were tested if they conform to the designed parts. The parts were then integrated into modules. The electrical and mechanical modules were tested. The modules were then integrated to form the system as a whole. The design was tested and if it conforms to the principle solution. The results from the system were obtained and compared with the requirements list. Some short falls were discovered and fed into the next cycle as part of the requirements list for the next phase of the development of the Cartesian robot for PCB drilling. Fig 1 is a diagrammatic representation of the process model described above. 


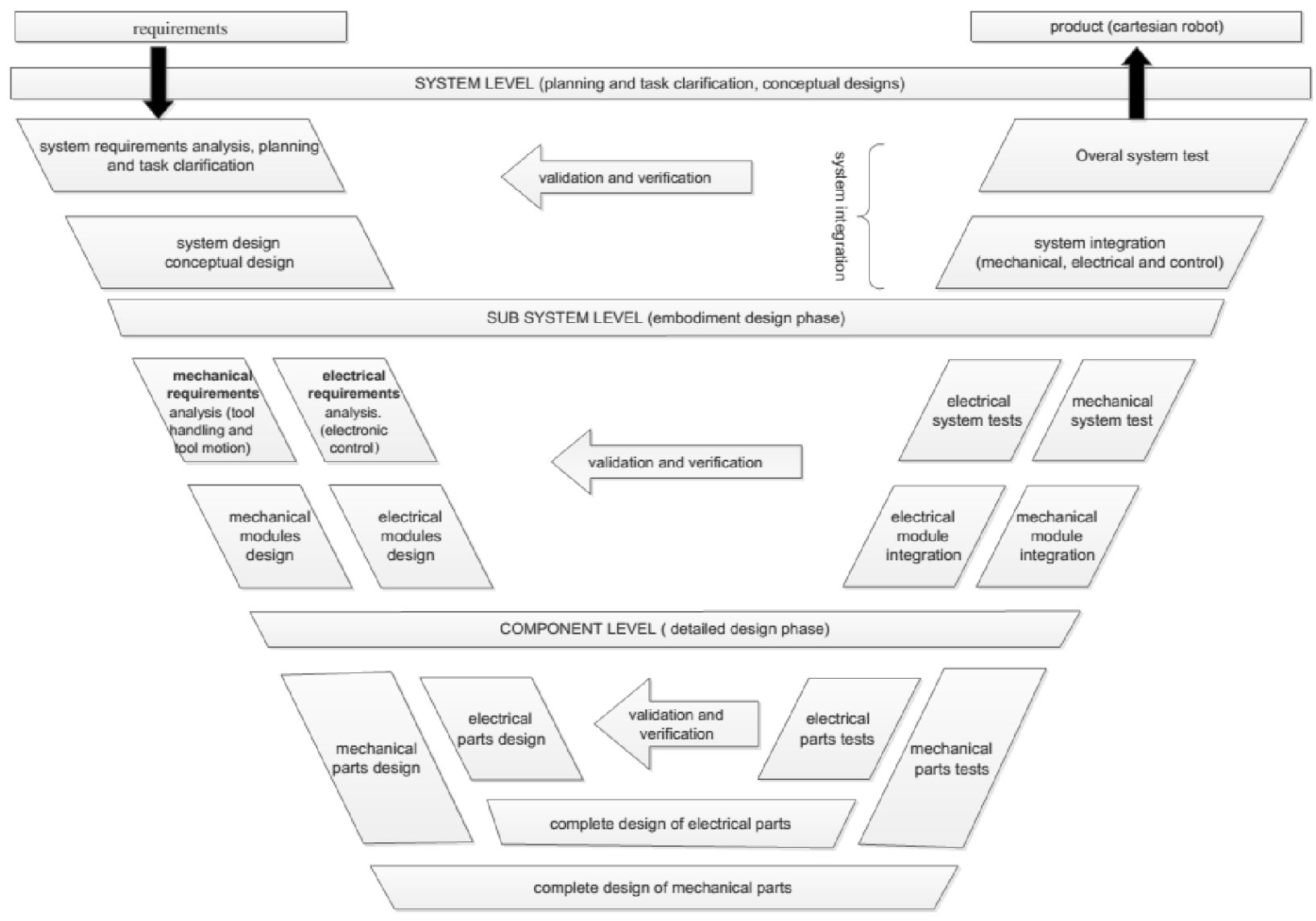

Figure 1: Process model for the development of a Cartesian robot for PCB drilling

\subsection{System development}

\section{System requirements}

Narrative requirements

Required:

- A system that will automatically extract coordinates of holes from a given gabber file, develops a path plan for the tool motion and execute the drill operation on the PCB.

Characteristics:

- Efficient energy consumption.

- $\quad$ Easy to operate. (it should be a plug and play system)

- Easy to maintain (with modules easy to isolate for repair and maintenance schedules)

- Work envelope of not more than the size of an A4 in the x-y plane.

- Portable such that it can be used as a desk machine.

- Must have a hard ware left and right tool compensation. (to minimize the number of sensors hence making maintenance easier.)

\section{Initial requirements}

Table 1: sample of Initial requirements list table

Abbr. Requirements 


\begin{tabular}{|c|c|}
\hline R1 & $\begin{array}{l}\text { Extract coordinates from a gabber file, communicates a signal that will move the tool piece in the } \mathrm{x} \text {, } \\
\mathrm{y} \text { and } \mathrm{z} \text { coordinates in the Cartesian plane, drill } 1 \mathrm{~mm} \text { through holes on PCB in a relatively less time }\end{array}$ \\
\hline $\mathrm{R} 2$ & Consumes minimum energy, and time in positioning the tool piece on the targeted coordinate. \\
\hline R3 & Simple maintenance. Made up of module that can be easily isolated and serviced in minimum time \\
\hline R4 & Drills holes on a single PCB at a time, and can store the drill files for future use \\
\hline R5 & The Cartesian robot should be portable and can be used a desk machine \\
\hline R6 & $\begin{array}{l}\text { The robot should be able to easily communicate with any computer through serial communication or } \\
\text { USB }\end{array}$ \\
\hline R7 & $\begin{array}{l}\text { Make use of materials that are readily available and can be processed locally (import only that cannot } \\
\text { be made locally) }\end{array}$ \\
\hline R8 & The robot must be working on a PCB that does not exceed A4 size \\
\hline R9 & The robot must make use of a dc system with a battery backup \\
\hline R10 & Hard wired tool compensation \\
\hline R11 & Manual loading of the tool piece on the spindle \\
\hline R12 & Use of serial communication, a usb to serial cable to be used in case of usb connections. \\
\hline R13 & Minimum achievable computer resolution \\
\hline R14 & Make use of prismatic joints \\
\hline
\end{tabular}

Below is a design requirements checklist [5]

Table 2:design requirements checklist table

\begin{tabular}{|c|c|}
\hline Main heading & Examples \\
\hline 1. Geometry & $\begin{array}{l}\text { Size, height, breath, length, diameter, space requirements, number, arrangement, } \\
\text { connections, extension }\end{array}$ \\
\hline 2. Kinematics & Type of motion, direction, velocity, acceleration \\
\hline 3. Forces & $\begin{array}{l}\text { Direction of force, magnitude of force, frequency weight, load, deformation, stiffness, } \\
\text { elasticity, stability, resonance }\end{array}$ \\
\hline 4. Energy & $\begin{array}{l}\text { Output, efficiency, loss, friction, ventilation state, pressure, temperature, heating, } \\
\text { cooling, supply, storage, capacity, conversion }\end{array}$ \\
\hline 5. Material & $\begin{array}{l}\text { Physical and chemical properties of the initial and final product, auxiliary materials, } \\
\text { prescribed materials (food regulations etc.) }\end{array}$ \\
\hline 6. Signals & Inputs and outputs, form, display, control equipment \\
\hline 7. Safety & $\begin{array}{l}\text { Direct safety principles, protective systems, operational, operator and environment } \\
\text { safety }\end{array}$ \\
\hline 8. Ergonomics & Man machine relationship, type of operation, clearness of layout, lighting, aesthetics \\
\hline 9. Production & $\begin{array}{l}\text { ensions, preferred production methods, } \\
\text { erances. }\end{array}$ \\
\hline 10. Qual & Possibilities of testing and measuring, application of special regulations and standards. \\
\hline 11. Assel & Spee \\
\hline 12. Transport & $\begin{array}{l}\text { Limitations due to lifting gear, clearance, means of transport (height and weight), } \\
\text { nature and conditions of dispatch }\end{array}$ \\
\hline 13. Operation & $\begin{array}{l}\text { Quietness, wear, special uses, marketing and destinations (e.g. sulphurous atmosphere, } \\
\text { tropical conditions.) }\end{array}$ \\
\hline 14. Maint & Servicing intervals (if any), inspections, exchange and repair, painting, cleaning \\
\hline 15. Recycling & Reuse, reprocessing, waste disposal, storage, \\
\hline 16. Costs & $\begin{array}{l}\text { Maximum permissible manufacturing costs, cost of tooling, investment and } \\
\text { epreciation }\end{array}$ \\
\hline 17. Schedules & velopment, project planning and schedules, delivery date \\
\hline
\end{tabular}


Table 3:: an extract of the incidence matrix table representing formal interaction between initial requirement and requirement checklist

\begin{tabular}{|c|c|c|c|c|c|c|c|c|c|c|c|c|c|c|c|c|c|c|}
\hline & & ${ }_{1}^{\mathrm{R}}$ & 2 & 3 & 4 & 5 & 6 & & & 8 & 9 & ${ }_{10} \mathrm{R}$ & ${ }_{11}^{R}$ & $12^{\mathrm{R}}$ & $13^{\mathrm{R}}$ & ${ }_{14}{ }^{\mathrm{R}}$ & $15^{\mathrm{R}}$ & $16^{\mathrm{R}}$ \\
\hline Function & ${ }_{1} \mathrm{C}$ & ${ }_{1}^{\mathrm{e}}$ & & & 1 & & 1 & & & & & & & & & & & \\
\hline Geometry & $2^{\mathrm{C}}$ & & & & & & & & & 2 & & & & & & & & \\
\hline Kinematics & $3^{\mathrm{C}}$ & & & & & & & & & & & & & & & $3^{\mathrm{e}}$ & & \\
\hline Forces & & & & & & & & & & & & & & & & $4^{e}$ & & \\
\hline Energy & & & 5 & & & & & & & & & & & & & & & \\
\hline Material & $6^{\mathrm{C}}$ & & & & & & & & & & & & & & & & & \\
\hline Signals & $7^{C}$ & $7^{\mathrm{e}}$ & & & & & & & & & & & & $7^{\mathrm{e}}$ & & & & \\
\hline
\end{tabular}

Using the initial requirements list and the requirements check list, an incidence matrix table was formulated to make sure that all the requirements are found in the design specification table. Table 4 is an extract of the design specification table for the Cartesian robot.

Table 4: an extract of the design specification table for the Cartesian robot

\begin{tabular}{|c|c|}
\hline Date & $\begin{array}{l}\text { Project: Cartesian robot for PCB drilling } \\
\text { 1. Functional performance: } \\
\text { Extracts coordinates from gabber file, calculates the } \\
\text { number of steps to be moved, move the tool piece to } \\
\text { the required coordinates, perform the drilling } \\
\text { operation }\end{array}$ \\
\hline & $\begin{array}{l}\text { Constrains } \\
2 . \quad \text { Geometry } \\
\bullet \quad \text { length } \leq 380 \mathrm{~mm} \\
\bullet \quad \text { width } \leq 290 \mathrm{~mm} \\
\bullet \quad \text { height } \leq 300 \mathrm{~mm} \\
\bullet \quad \text { Length } \leq 290 \mathrm{~mm} \\
\bullet \quad \text { Width } \leq 210 \mathrm{~mm} \\
\text { Work piece (work envelope) } \\
\text { • } 1 \mathrm{~mm}\end{array}$ \\
\hline & 3. $\frac{\text { Kinematics }}{\bullet \quad \begin{array}{l}\text { Prismatic joints, to offer translation in the } \mathrm{x}, \mathrm{y} \text { and } \mathrm{z} \\
\text { axis }\end{array}}$ \\
\hline & $\begin{array}{l}\text { 4. } \quad \text { Energy } \\
\text { • Uses minimum electrical energy }\end{array}$ \\
\hline & $\begin{aligned} \text { 5. } & \text { Material } \\
\bullet & \text { Make use of locally available material } \\
\bullet & \text { Easy to work on materials } \\
\bullet & \text { Copper clad PCBs }\end{aligned}$ \\
\hline & 6. Signals \\
\hline
\end{tabular}




\begin{tabular}{|l|l|}
\hline & $\bullet$ Industrial standards \\
& - $4-20 \mathrm{~mA}$ and \\
& - $0-5 \mathrm{~V}$ to be used as control signals. \\
& - Serial communication, and USB \\
\hline
\end{tabular}

\subsection{Principle solution}

An analysis on the system was made and from the design specifications a principle solution was developed. The principle solution shows how the system will work in principle. It also shows all the necessary components and how they are linked. Below is a block diagram to illustrate the principle solution. A computer will be used to develop the PCB layout design. From the PCB layout design, a set of coordinates is generated and through a communication protocol, it is communicated to the controller which will in turn coordinates the actuator movements. Position sensors will be used to determine the position of the tool piece and give a feedback to the controller for the appropriate action to be taken.

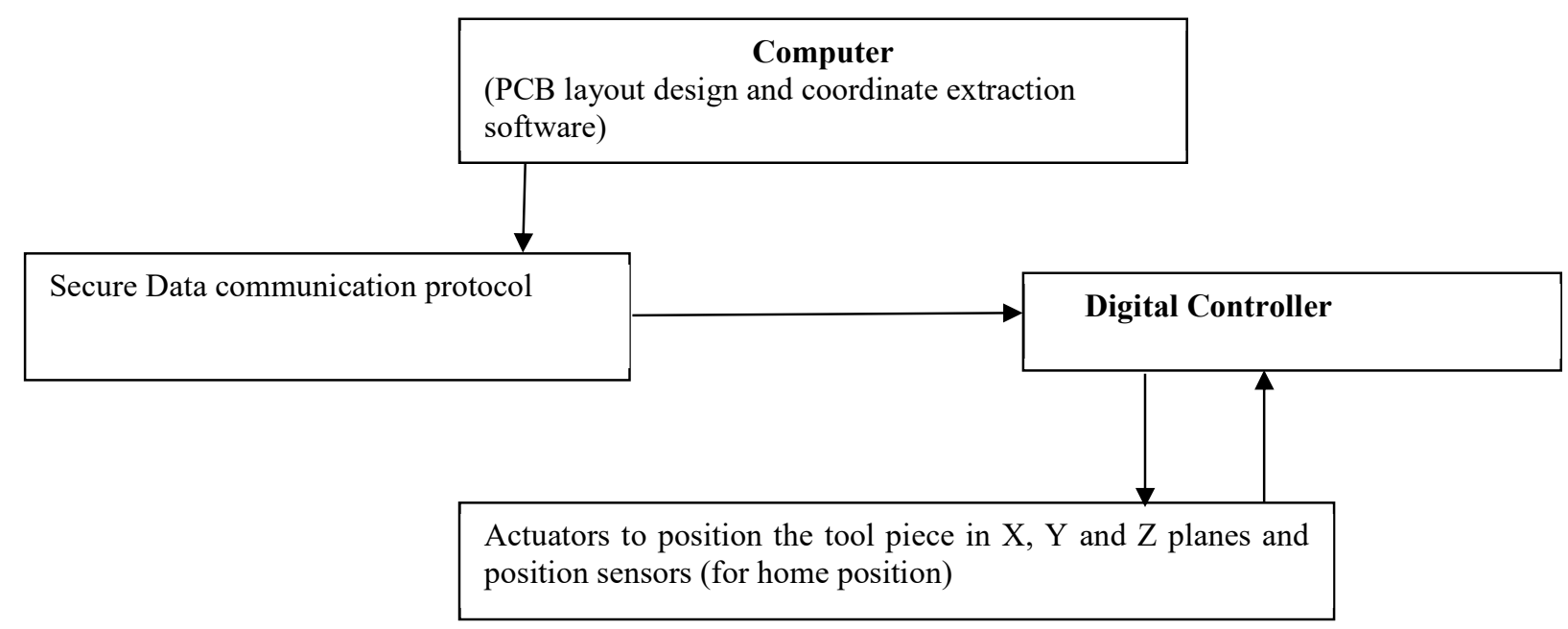

Figure 2: principle solution for the Cartesian robot for PCB drilling

\subsection{Domain Specific Design}

\section{Chassis Design (Mechanical Domain)}

The chassis was designed by making use of prismatic joints. Prismatic joints will give enough support for the tool piece and work piece weight. Various configurations were looked at and evaluated as follows. The generated concepts will be looking on the movement of the work piece and tool piece.

\section{Concept 1: Tool piece and work piece moving}

This mechanism the horizontal work table represents the y axis movement which is done by positioning the work piece to achieve the y coordinate. The vertical part where the drill is assembled is used to first locate the $\mathrm{x}$-axis coordinate by positioning the drill then finally moving the drill in the z-axis that is vertically up and down to execute the drilling processes. With this mechanism, the work piece will not be stationary therefore it will be moving. Therefore, the coordinates of the point to be drilled will be defined with the motion of work piece (y-axis) and that of the tool piece (x-axis). Then the drilling process is executed by moving the drill up and down (z-axis). The Fig below illustrates the above explanation. 


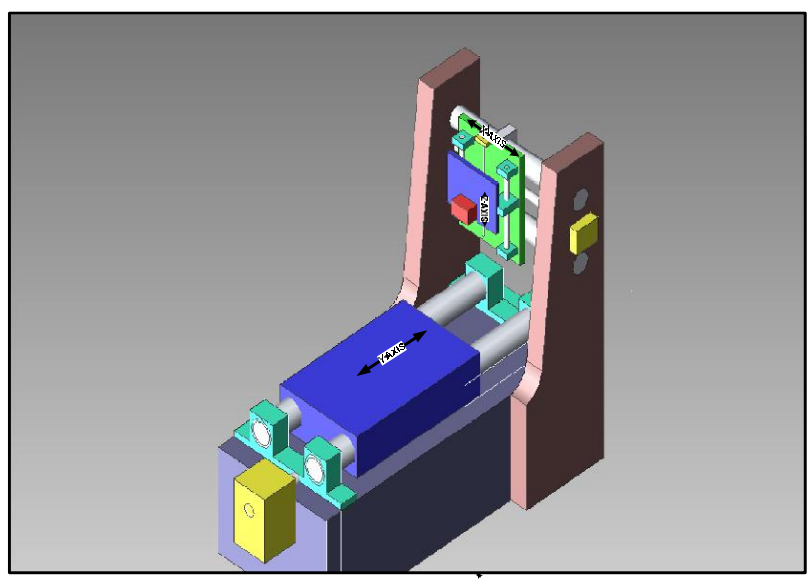

Figure 3: A diagrammatic representation of the concept 1

Advantages

- Quickly arrives to the desired coordinate because the is simultaneous movement in the $\mathrm{y}$ and $\mathrm{x}$ axis. Disadvantages

- Relatively larger work envelope in moving work piece.

- Complexity of programming and execution of commands.

Concept 2: Work piece moving and tool piece stationery.

In this concept, the frame holding the work piece will be moving in the $\mathrm{y}$ and $\mathrm{x}$ axis. The tool piece will be stationery relative to the $\mathrm{y}$ and $\mathrm{x}$ axis and only moves in the $\mathrm{z}$ axis

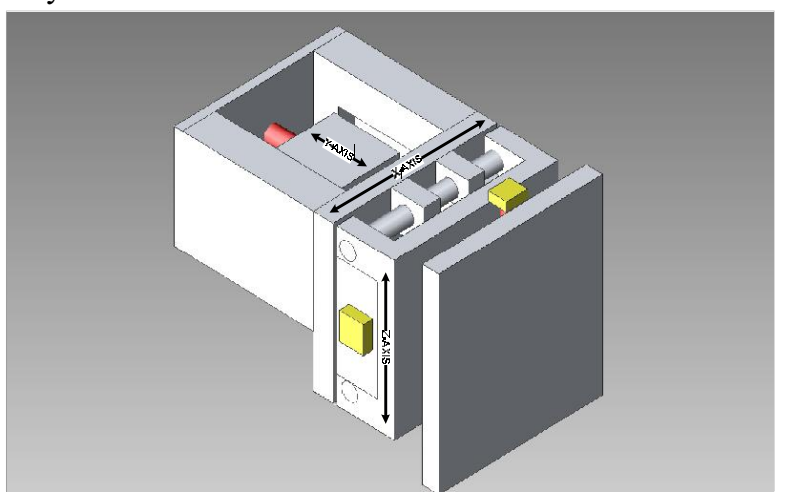

Figure 4:diagramatic illustration of concept 2

Advantages

- Tool positioning is achieved

- If the frame holding the work piece is light it means less energy is invested to locate the coordinate Disadvantages

- Positioning is complex

- The work envelope is relatively big and the design requires big space 


\section{Concept 3: Tool piece moving}

In this concept the tool piece moves in all the three axis. The work piece will be stationery.

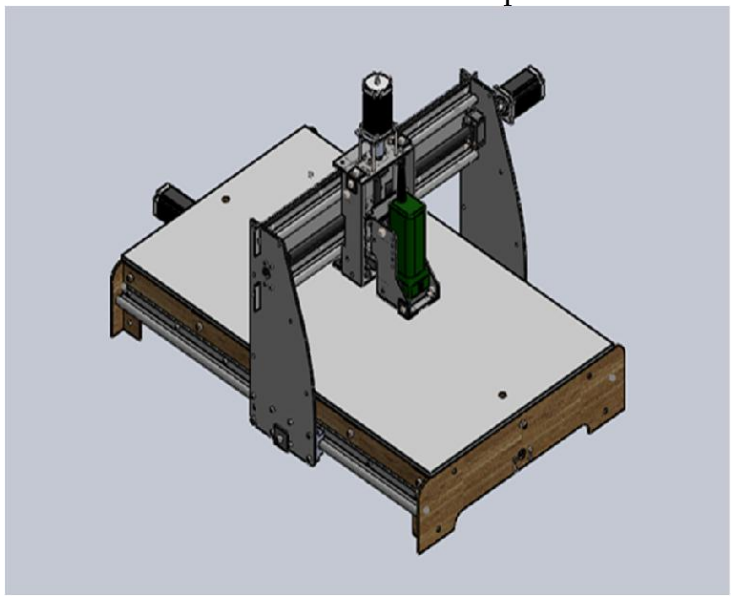

Figure 5: illustration of concept 3

Advantages

- High precision in tool positioning.

- Less work space requirements.

Disadvantages

- $\quad$ More energy is required for the y axis movement

After analyzing the concepts concept 3 was chosen because of the minimum space requirements and easy and accurate positioning of the tool piece on the desired coordinate. It is also easy to compensate for the tool in the $\mathrm{x}$ and y axis with the concept 3 .

\section{Electronics domain}

\section{Hardware development}

Open hardware components were used for the system, because of their modularity for ease of maintenance and troubleshooting in case of a fault. Arduino Uno development board was used. A CNC shield for stepper motor driving was used. Stepper motors were used as the actuators because of their ability to be used in open loop control systems. This will reduce on cost and complexity of the system.

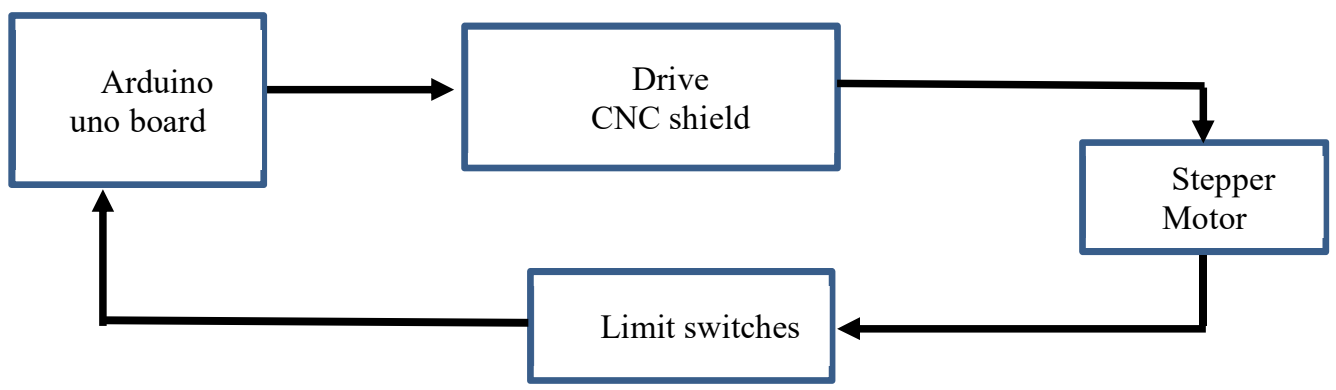

\section{A layout of the electronics components.}

\section{Software development}

VB 2013 was used to develop an algorithm that extracts coordinates from the gabber file and load them on the com port. The Arduino code will then calculate the number of steps required to move in the $\mathrm{y}$ axis and the $\mathrm{x}$ axis. It then 
commands the stepper motors to move in the respective axis the respective calculated distance. The system will use a point to point path plan.

\section{System development and integration}

The hardware components were integrated and tests were carried out to ascertain if the developed systems were confirming to the designs. Modular tests were done followed by system testing.

Proteus software was used to develop the PCB layout. The software generated a gabber file and stored it in a folder as a text file. VB software was used to extract the coordinates from the text file and load then on a port to communicate with the Arduino Uno (the controller) using a secure USB communication protocol.

The system was developed with an option to manually add the coordinates, in case one fails to generate the text file for the coordinates of the holes. Below is a GUI for developed from VB.

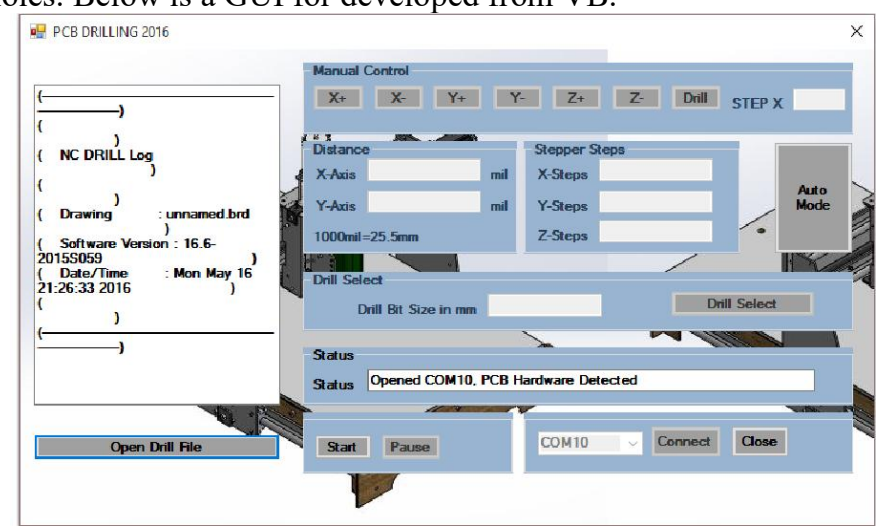

Figure 6: GUI for the Cartesian robot

Below are pictures of the design during assembly and the $3 \mathrm{D}$ drawing of the design.

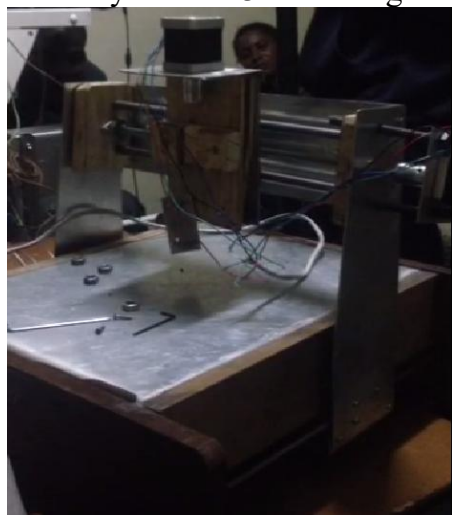

Figure 7:Cartesian robot during assembly 


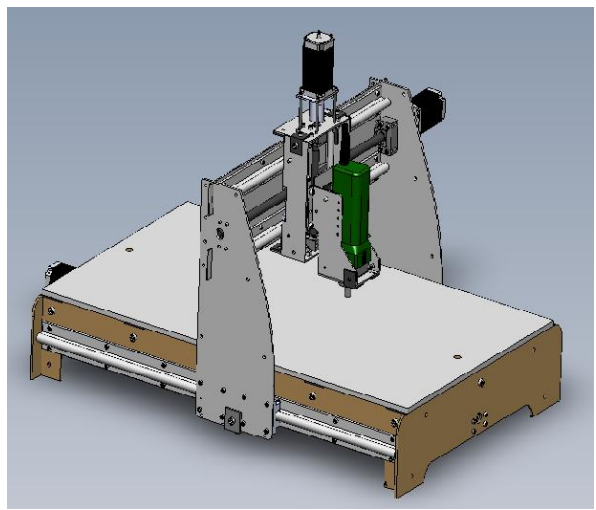

Figure 8:3D drawing of the Cartesian robot

The Cartesian robot have a resolution of $0.021 \mathrm{~mm}$ per step, which means the minimum distance that can be moved in any of the axis is $0.021 \mathrm{~mm}$.Considering that the minimum hole diameter is $1 \mathrm{~mm}$ our resolution is good and acceptable. It is also critical to note that we have to keep our grid snap tool at values above $0.021 \mathrm{~mm}$ when designing the PCB layout.

The maximum current drawn by the system is $3 \mathrm{~A}$ and the power rating for the system is $72 \mathrm{~W}$. A $24 \mathrm{~V}$ battery can be used as a backup system which makes the system more user friendly.

\section{Conclusion}

A Cartesian robot for PCB drilling was developed which is fast and accurate in drilling of holes on PCBs. The robot's chassis was developed from wood and steel, which are cheap materials but with required specifications. An Arduino Uno development board was used as the controller of the system, a CNC shield was used as the driver for the stepper motors. This configuration brought about the modularity of the system, which will give us ease of troubleshooting and maintenance. A secure USB communication protocol was used to communicate between the control unit and the master computer.

The system is more suitable for research institutions and academic institutions where there is mass production of PCBs. The designed robot is flexible and can accommodate various PCB designs. Teaching of courses in CAD/CAM is achievable using the developed robot and tool path motion can easily be demonstrated.

\section{Recommendations}

For future work a feedback system is to be developed that will verify that the tool position is on the same coordinate as the hole to be drilled. The system should then use automatic tool compensation using the adaptive control algorithm. The feedback system may be based on machine vision (because the routing is done using another system).

Gear mechanisms can also be considered to increase the speed and machine resolution. Once machine resolution is high the system can be upgraded into both a PCB routing and drilling machine. This machine will reduce the processing time significantly by eliminating the chemical etching process. 


\section{References}

[1] V. S. Vasić and M. P. Lazarević, "Standard Industrial Guideline for Mechatronic Product Design," FME Transactions, vol. 36, no. No 3, pp. 103-108, 2008.

[2] R. B. A. Rahman, U. Pulm and R. Stetter, "Systematic Mechatronic Design of a Piezo-Electric Brake," in International Conference on Engineering Design, ICED'07, Paris, August 2007.

[3] VDI Guideline 2206, "Design Methodology for Mechatronic Systems," VDI, Dusseldorf, 2004.

[4] Y. Verbelen, D. Van Belle and J. Tiete, "Experimental analysis of small scale pcb manufacturing techniques for fablabs.," nternational Journal of Engineering Innovations and Research, vol. 2, no. 2, p. 136, 2013.

[5] G. Pahl, W. Beitz, J. Feldhusen and K. H. Grote, Engineering Design: A Systematic Approach 3rd edition, K. W. a. L. Blessing, Ed., London: Springer-Verlag, 2007. 